\title{
Celebrating Outstanding Science at the European Academy of Dermatology and Venereology's 30th Anniversary Congress
}

Authors:

Citation:
Asli Bilgiç, ${ }^{1}$ Ivan Bogdanov, ${ }^{2}$ Paola Pasquali, ${ }^{3}$ Mariano Suppa, ${ }^{4}$ Marie-Aleth Richard, ${ }^{5}$ Dedee F Murrell6,7

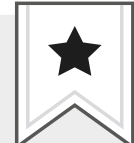

1. Department of Dermatology and Venereology, Akdeniz University, Faculty of Medicine, Antalya, Turkey

2. Department of Dermatology and Venereology, Acibadem Cityclinic Tokuda Hospital, Sofia, Bulgaria

3. Dermatology Department, Pius Hospital de Valls, Tarragona, Spain

4. Department of Dermatology, Hôpital Erasme, Université Libre de Bruxelles, Brussels, Belgium

5. Department of Dermatology, University Hospital of La Timone, Marseille, France

6. Department of Dermatology, St George Hospital, Sydney, Australia

7. Faculty of Medicine, University of New South Wales, Sydney, Australia

${ }^{*}$ Correspondence to d.murrell@unsw.edu.au

EMJ Dermatol. 2021;9[1]:24-28.
THE ANNIVERSARY edition of the European Academy of Dermatology and Venereology's (EADV) $30^{\text {th }}$ Congress was held between $29^{\text {th }}$ September and $2^{\text {nd }}$ October 2021. As COVID-19 remains a barrier to global travel, the EADV's 30 th Anniversary Congress was staged virtually, providing access to the latest research, breakthroughs, and scientific advances to dermato-venereology professionals.

The ever first EADV Congress took place in Florence, Italy, in 1989 when the EADV was just 2 years old. It was hosted by the Academy's first ever President, Emiliano Panconesi. At that time, the EADV welcomed a small number of delegates, which grew to over 1,000 in just 2 years. The $29^{\text {th }}$ EADV Virtual Congress in 2020 was the first to be organised in an all-virtual format.

Celebrating three decades of outstanding science, the 4-day scientific programme of the EADV's $30^{\text {th }}$ Anniversary Congress, was fully packed with plenary lectures by renowned scientists; late breaking news sessions, with analysis of the latest published data; clinical updates; case discussions relevant for daily practice; and the latest trends in dermato-venereology, which were delivered by over 550 leading experts in 160 simu-live sessions and 11 keynote lectures from thought leaders. The congress attracted over 9,000 participants from 120 countries.

The EADV is one of the most prominent and influential academies in the field of dermatovenereology. As a non-profit organisation with nearly 7,000 members across 116 different countries, the EADV provides a valuable service for every type of dermato-venereologist professional. It has re-established its activities as virtual meetings and webinars, online tools and innovative e-learning offerings, and enhanced its online communication by putting access to digital learning at the centre of its education strategy as a response to the COVID-19 pandemic.

\section{ADVOCACY}

The EADV is also taking a leading role in promoting dermatology and venereology in European Union (EU) health policy to support the specialty to deliver better patient care. The EADV has identified two high priorities on the EU policy agenda for proactive advocacy: the new Europe's Beating Cancer Plan and Horizon Europe initiatives. 
The first focus is on Europe's Beating Cancer Plan, which is mainly addressing priorities in skin cancer prevention and early detection. For this purpose, there have been a series of Policy Roundtables to create a patient centred approach to shape policies that support public awareness of skin cancer risk factors and help with prevention.

\section{Europe's Beating Cancer Plan}

The EADV welcomes Europe's Beating Cancer Plan, which includes four main pillars: prevention, early detection, diagnosis and treatment, and survivorship. All are highly relevant for patients with skin cancer such as melanoma and nonmelanoma skin cancers. Furthermore, the EADV also supports the efforts to prevent human papillomavirus (HPV)-induced cancers such as the HPV vaccination programme.

\section{SKIN CANCERS}

Skin cancer is one of the most common cancers in the EU. In 2020, over 106,000 EU citizens were diagnosed with different forms of skin cancer, and more than 20,000 Europeans die from it annually. The cost of care for patients with skin cancer in European countries is estimated at 2.7 billion EUR annually and, because of this cost, the disease is also a considerable economic burden to healthcare systems. Thus, skin cancer was one of the important focuses of the EADV $30^{\text {th }}$ Congress.

Harald Kittler, Medical University of Vienna, Austria, discussed what is 'atypical' when speaking about pigmented skin lesions. The term 'atypical' is used to indicate the relationship of a nevus to melanoma by indicating a greater risk of becoming melanoma. He suggested that a lesion should not be called 'atypical' as some think it has a higher risk of transformation; however, some 'typical' looking lesions can transform into melanomas. The suggested action is to follow the three-rule advice if you are uncertain: correlate (the dermoscopic image with the clinical one); compare (the dermoscopic images of all the pigmented lesions); and control (do a follow-up).

Furthermore, Rainer Hofman-Wellenhof, Medical University of Graz, Austria, spoke on the dermoscopic indicators for early melanomas: an atypical network, regression of more than $50 \%$, irregular hyperpigmented areas, angulated lines, and prominent skin markings.

Philipp Tschandl, Medical University of Vienna, Austria, presented an in-depth update on the use of artificial intelligence (AI) in dermatology. He gave insights into the use of $\mathrm{Al}$ in diagnosing, covering with no images; with clinical images; with dermoscopic images; in follow-up of images; in full-body photography; and finally in histology specimens. Limitations of Al models when it comes to precise and accurate detection were discussed.

The advantages and disadvantages of telediagnosis were discussed by Peter Soyer, The University of Queensland, Australia, and founder of the International Society of Teledermatology (ISTD). He explained how the pandemic has impacted cancer diagnoses in the field of dermatology and other clinical fields. In Australia, there have been 145,000 less mammograms undertaken for screening breast cancer and up to 38\% less early diagnoses of this disease. He further emphasised that healthcare professionals must rethink their ways of working in regard to their specialty, as there is now a new era, where every day work is getting closer to technological advances.

Despite concerns, the number of skin cancer detection apps available for consumer use is growing. Lloyd Steele et al. from Queen Mary University of London, UK, and the Blizard Institute shared the results from their study, which assessed the performance metrics of two published machine-learning models for Merkel cell carcinoma and amelanotic melanoma. Both have reported an expert-level performance and one is already being sold directly to the public.' They further suggested that the current approval of models to the public, without any reported performance metrics for rare but potentially life-threatening skin cancers, is ethically questionable, while the question of sufficient accuracy for deployment remains an unanswered one. Thus, there must be more transparency around the safety and efficacy of these apps. These machine-learning models have potentially negative consequences at both a personal and societal level and could put lives at risk. 


\section{SQUAMOUS CELL CARCINOMA}

Squamous cell carcinoma (SCC) is one of the most common skin cancers and belongs to nonmelanoma skin cancers group. Surgery remains the best option for treatment if complete removal is feasible, with acceptable morbidity. Ricardo Vieira, Dermatologist from Coimbra University Hospital, Portugal, discussed surgery in advanced SCC. The advanced SCC can be defined as a large tumour, invading tissues beyond subcutis, with poor differentiation, perineural spread, and located on chronic inflammatory conditions such as burn scars and chronic ulcers. Surgical margins should be at least $10 \mathrm{~mm}$ and microscopically controlled surgery should be performed when feasible and available. Flap repairs are not recommended if a tumour-free margin cannot be assured, and a delayed reconstruction is recommended in those cases. Microscopically controlled surgery has a better clearance of the tumour, and it is the best approach to removing tumours with perineural invasion; however, the fact that the frozen sections are difficult to interpret still have to be taken into account, and the procedure is limited to tumours with no extension to deep structures.

\section{MELANOMA}

Eduardo Nagore, Instituto Valenciano de Oncolgía, Valencia, Spain, discussed Mohs surgery and its areas of usage. He emphasised that Mohs surgery has been proved to be an excellent choice for the treatment of lentigo maligna (LM) and LM melanoma. However, it is uncertain if all head and neck LM/LM melanoma are candidates for this surgical technique, or only those with specific characteristics such as lesions with ill-defined borders or tumours with a severe sun-damage background. The use of Mohs surgery is promising in some of the acral lentiginous melanomas such as nail unit melanomas, which are difficult to operate due to their specific anatomical characteristics. However, its use remains with doubtful usefulness in lesions located on the soles. For malignant melanomas located on the trunk and the extremities, there is no proven benefit from the use of micrographic surgery.
In regard to optimising Mohs surgery procedures, Cristian Navarrete-Dechent, Pontificia Universidad Católica de Chile, Chile, further discussed imaging techniques. As tumours that are candidates for Mohs surgery are usually associated with subclinical extensions, the most commonly used imaging techniques are widefield images (e.g., CT and MRI), which are not the best tools for microscopic and subclinical diseases. He suggested that the combination of reflectance confocal microscopy and optical coherence tomography imaging may be excellent tools for a real-time, non-invasive, comprehensive $3 \mathrm{D}$ sampling in vivo and improve the diagnostic accuracy and margin assessment of basal cell carcinoma and LM prior to Mohs surgery.

\section{HUMAN PAPILLOMAVIRUS INFECTION}

HPV is the most widespread and common sexually transmitted disease worldwide, with more than $80 \%$ of sexually active women and men expected to acquire at least one HPV infection by the age of 45 years. They manifest as genital warts (condyloma acuminata), which are soft fleshy growths that usually develop around the genitals or anus. HPV is also pre-cancerous for various cancers including cervical cancer, penile cancer, anal cancer, and in some cases of oral and throat cancer as well as others.

Researchers from Denmark (Pernille Lindsø Andersen et al., Næstved Hospital) have uncovered a link between genital warts and the human leukocyte antigen system, which is part of the genetic region that holds genes essential for normal functioning of immune response, helping to distinguish between 'foreign items' called antigens (which cause the body to make an immune response). Their findings suggest that genetic alterations in the human leukocyte antigen system influence the risk of genital warts. They identified 12 protective gene variations (odds ratios: 0.4-0.8), in the preliminary cohort and seven risk alleles (odds ratio: 1.1-1.3) in the present cohort. Individuals with risk alleles were less successful at recognising the HPV virus and, therefore, more likely to present with genital warts. Conversely, participants with protective alleles had better immune responses and were more effective at recognising HPV. 


\section{VACCINES}

Steve Pascolo, University of Zurich, Switzerland, trained as an immunologist at the Pasteur Institute, Paris, France, presented the past (initial preclinical and clinical studies of vaccines based on synthetic mRNA, characteristics), the present (synthetic mRNA vaccines against COVID-19), and the future of mRNA vaccines (e.g., design of anticancer vaccines). He also went well beyond their use as vaccines discussing the great potential of synthetic mRNA in medicine. Synthetic mRNA vaccines have proven, as a result of the COVID-19 pandemic, to be fast and easy to produce as well as safe and efficient. This format is now expected to be largely exploited to create new vaccines (against infectious agents as well as against cancer), and also to replace previously existing vaccines that presented challenges in production, storage, efficacy, and side effects.

\section{BIOLOGICS, INFECTIONS, AND VACCINES}

Biologics are increasingly used for psoriasis. They are often thought to be associated with an increased risk of infections. However, different risks were reported in previous studies. COVID-19 has resulted in attention for risk of serious infections $(\mathrm{SI})$ and respiratory tract infections (RTI) in this population. Lara van der Schoot et al., Radboud University Medical Center, Nijmegen, the Netherlands, showed the results of their study, ${ }^{2}$ which evaluated the differential effect of biological therapies on risk of SI and RTI, including severe acute respiratory coronavirus 2 infections among patients with psoriasis who were treated with currently available biologics in a real-world setting. The study included 714 patients with 1,325 treatment episodes. Adjusted analyses showed no differential risk of SI or RTI between adalimumab, etanercept, infliximab, ustekinumab, secukinumab, ixekizumab, and guselkumab in a daily practice cohort of patients with psoriasis.

\section{THE BURDEN OF SKIN DISEASE}

Little is known about the prevalence of skin diseases in the general population across Europe. Moreover, there is a lack of solid, objective, and homogeneous data at the European level on the impact skin diseases have on quality-of-life, including stigmatisation, or on the perception of and access to dermatologists.

The EADV has, therefore, commissioned an adult population-based survey. The Burden of Skin Disease in Europe evaluated the prevalence of dermatologic or venereological problems across Europe and documented the reasons for consulting a dermatologist, impact of skin disease on patients, public perception of skin diseases, skin disease care pathways, prescribed treatment, and confidence in dermatologists. Marie-Aleth Richard, University Hospital of La Timone, Marseille, and EADV Board Member, led the survey, and shared preliminary results of the study, which is the largest ever undertaken of its kind in Europe. Data has now been collected from 44,689 adults from 27 European countries, as well as the UK and Switzerland. Preliminary findings show that among 21,401 members of the general population, $47.9 \%$ of people 18 years of age or older self-reported at least one skin condition. The most common skin condition among those surveyed is fungal skin infection, affecting almost one in 10 people (9.07\%). Other common conditions, each affecting more than one in 20 people, were atopic dermatitis (eczema; 5.34\%), alopecia (5.22\%), and acne (5.49\%). Furthermore, skin symptoms or unpleasant skin sensations, including tightness and itchiness, as a specific consultation requests were reported by $20 \%$ of people aged 18 years or older. The study findings after full-data analysis can act as a guiding light to have a firm understanding of the burden of skin diseases on patients and the healthcare systems that further guide the health policies.

Although the congress might have ended, content remains accessible to registered delegates to watch on demand. The scientific content remains available 3 months after the congress, and EADV members have access to the content of previous congress and symposiums.

Healthcare professionals are all waiting to meet personally in the future as personal interactions are an essential part of scientific discussions. However, moving beyond the COVID-19 pandemic era, the EADV plans to retain, enhance, and build upon many of the positive innovations that helped the organisation to adapt their offerings 
for healthcare professionals and patients during that time. Virtual activities will continue to be an important component of future EADV scientific events, allowing for broader attendance and a greater diffusion of knowledge to help members both optimise patient care and deliver the advances made in clinical research in the many different fields.

\section{References}

1. Steele $L$ et al. Do Al models recognise rare, aggressive skin cancers? An assessment of a direct-to-consumer application in the diagnosis of Merkel cell carcinoma and amelanotic melanoma. J Eur Acad Dermato
Venereol. 2021;DOI:10.1111/jdv.17517.

2. Van Der Schoot $L$ et al. Risk of respiratory tract infections and serious infections in psoriasis patients treated with biologics: results from the BioCAPTURE registry. Available at: https://s3.eu-central-1. amazonaws.com/cst.eadv/eadv2021/ abstracts/24432.html.pdf. Last accessed: 21 October 2021. 